\title{
PROMOTION OF MENTAL HEALTH LITERACY IN ADOLESCENTS: A SCOPING REVIEW PROTOCOL
}

\author{
Joana Rita Pimenta Nobre ${ }^{1}$, Carlos Alberto da Cruz Sequeira², Carme Ferré-Grau ${ }^{3}$ \\ ${ }^{1}$ Nurse; PhD Student in Nursing and Health at the University of Rovira i Virgili (Tarragona - Spain) \\ ${ }^{2} \mathrm{PhD}$; Coordinator Professor at the Nursing School of Porto (Portugal) \\ ${ }^{3} \mathrm{PhD}$; Coordinator of the Doctoral Program in Nursing Sciences at the Faculty of Nursing - University of Rovira i Virgili (Tarragona - \\ Spain)
}

Correspondence: Joana R.P. Nobre, joana.r.nobre@gmail.com

Funding / Conflicts of interest: There is no funding or conflict of interest for this project. This scoping review will be developed under the PhD Program in Nursing and Health of the University of Rovira i Virgili (Tarragona - Spain).

\begin{abstract}
Introduction: Considering the increasing and significant prevalence of mental health problems in today's society and in particular adolescents, as well as the perspectives pointed to the future by the World Health Organization, it is important to know and synthesize the evidence currently available regarding programs/interventions promoters of mental health literacy in adolescents.

Aim: The aim of this review is to map available evidence on programs/interventions to promote mental health literacy in adolescents in school context.

Inclusion criteria: Will be included published and unpublished primary and secondary studies on programs/interventions to promote mental health literacy in adolescents in school context, written in Portuguese, Spanish or English, between 2013 and 2019.

Methods: A scoping review protocol was developed according to the guidelines of the Joanna Briggs Institute and the PRISMA-SCR. From the objective and the questions of the review, the databases and repositories for the research were selected to identify the studies that meet the eligibility criteria. The selection of articles, data extraction and synthesis will be performed by two reviewers independently, using an instrument created by the reviewers based on the Joanna Briggs Institute model.
\end{abstract}

Keywords: Adolescents, Mental Health, Health Literacy; Nursing. 


\section{Introduction}

In Portugal, as in the world, there is a huge prevalence of mental disorders in the general population as well as in adolescents and young people ${ }^{1,2}$.

Nowadays, it is known that mental disorders account for $12 \%$ of diseases worldwide, and in developed countries the figure rises to $23 \%^{3}$. According to the World Health Organization ${ }^{4}$, it is estimated that by 2020 about $15 \%$ of diseases will be behavioral or mental, and one in five adolescents may develop a mental disorder. Also Kelly, Jorm \& Right $^{5}$ state that $50 \%$ of people who will suffer from a mental disorder will experience their first episode before age 18 , and according to $\mathrm{WHO}^{6}$ this may occur even before 14 years.

The literature, so far, tells us that most Portuguese people have a problematic or inadequate health literacy level $^{7}$ and that, specifically, adolescent mental health literacy levels are modest ${ }^{8,9}$. The concept of mental health literacy is not recent, having been defined in 1997 by Jorm et $\mathrm{al}^{10}$ as the knowledge and beliefs about mental disorders that aid their recognition, management and prevention. The low level of literacy in adolescent mental health is a problem in current Portuguese society, which contributes to the lack of adolescent help seeking, affects their development and increases the risk of recurrence of psychiatric disorders ${ }^{11}$.

In recent years, researchers in the field of mental health have shown growing interest in the positive dimension of mental health over the disease-centered dimension, arguing that strengthening people's mental health is a protective factor as it promotes their resilience as adaptive capacity ${ }^{12}$, entering in the field of positive mental health. This promotion of the salutogenic dimension of mental health should start as early as possible in the life cycle, and adolescence is a vital opportunity period to promote mental health ${ }^{13}$, as better literacy at a young age is direct impact on adulthood, enabling adolescents to acquire knowledge and define behaviors that will accompany them in their future life ${ }^{14}$, namely the ability to positively manage their thoughts and emotions, to build social relationships and healthy family members, all based on a strong positive sense of identity.

Thus, the WHO defined in the Mental Health Action Plan 2013-202013 that one of the objectives worldwide is to implement strategies for the promotion and prevention of mental health, alerting to the importance of intervening not only in needs of people with mental disorders but also in the protection and promotion of the mental health of all citizens.

Given the significant prevalence of adolescent mental health problems as well as the prospects for adolescent mental health problems, it is necessary to know and summarize the evidence currently available regarding adolescent mental health literacy programs / interventions.

To this end, it was specifically decided to conduct a scoping review, as it considered the most appropriate methodology, since this type of review aims to: map existing evidence in relation to a particular area or topic; assess the reliability, relevance and potential costs of performing a systematic literature review; provide a synthesis of research results and disseminate them; and identify potential gaps in existing literature ${ }^{15,16,17}$.

A preliminary exploratory research on this subject was carried out, during September of 2019, in the JBI Database of Systematic Reviews and Implementation Reports, Cochrane Database of Systematic Reviews, CINHAL and MEDLINE (via EBSCO), and it was found a systematic review in this area developed by Morgado \& Botelho ${ }^{14}$, where articles were searched between 2008 and 2013, in the scientific databases CINHAL and MEDLINE, which resulted in 3 articles (one from 2008, one from 2009 and one from 2012). The mentioned systematic review ${ }^{14}$ found evidence that mental health literacy interventions are: the cognitive behavioral intervention in the school context, the psychoeducation intervention as well as the mental health education intervention in the school context, all aimed at adolescents without mental disease. In addition, it also found evidence that identifies the school context as a field in this area. 
The scoping review proposed in this protocol will differ from the previous review because it will be more comprehensive with respect to the research and will be more up to date, so it was decided to depart from this systematic review and to conduct the research from 2013, in a broader way to include available published and unpublished evidence on this topic.

The scoping review we intend to be carried out, following the methodology proposed by the Joanna Briggs Institute ${ }^{17}$, aims to map the available and most recent evidence on programs/interventions to promote mental health literacy in adolescents in a school context.

\section{Review questions}

This review aims to answer the primary question:

RQ 1. What is known in the existing literature on programs /interventions to promote mental health literacy in adolescents in a school context?

In addition, some secondary questions were formulated that will guide the review:

RQ 2. What are the characteristics of programs/interventions to promote mental health literacy in adolescents evidenced in the literature?

RQ 3. In which environments/contexts are these programs/interventions carried out?

RQ 4. What are the barriers and facilitators for the implementation of these programs/interventions?

\section{Inclusion criteria}

- Types of participants - The review will include studies whose participants are adolescents aged 10-19 years, as we adopt the WHO $(2012)^{6}$ definition of adolescents. Note that adolescents included in the studies cannot have diagnosed mental illness.

- Concept - Regarding the concept, will be included studies on programs/interventions to promote mental health literacy, which meets the objective defined for this review.

- Context - Regarding the context, this review will accept studies that include adolescents in the school context ( 2 nd and 3 rd cycles of primary education and secondary education).

- Types of studies - This review will include published and unpublished primary and secondary studies to gain access to a wider range of available information; in terms of language, studies that are written in Portuguese, Spanish or English, as they are the languages that the reviewers domain; and in temporal terms, studies published between 2013 and 2019, so that only articles with the most recent evidence are included.

\section{Methods}

The study is intended to be a scoping review, which will methodologically follow Joanna Briggs Institute guidelines ${ }^{17}$ and will also follow the Preferred Reporting Items for Systematic reviews and Meta-Analyses extension for Scoping Reviews checklist (PRISMA-ScR) ${ }^{18}$ for the presentation of the review report.

- Search strategy - Comprehensive research will be carried out on the MEDLINE with Full Text, CINHAL Plus with Full Text, SciELO and SCOPUS scientific databases, as well as the OpenGrey (European Repository) and RCAAP (Open Access Scientific Repository of Portugal) scientific repositories. 
The descriptors selected for the search in these databases are MeSH (Medical Subject Headings) terms and DeCS (Health Science Descriptors) terms: adolescent*, mental health, literacy, health literacy, program*, nursing.

Will be used the research strategy recommended by the Joanna Briggs Institute ${ }^{17}$, which consists of three stages: 1) a limited initial search will be performed on two databases (MEDLINE with Full Text e CINHAL Plus with Full Text) with the Boolean phrase 'adolescent*' AND 'mental health'AND 'literacy' AND 'health literacy' AND 'program*' AND 'nursing', followed by an analysis of the terms used in the titles and abstracts of the articles found in order to identify all relevant relevant terms and to determine the final Boelan phrase; 2 ) in the second step, the search will be performed in all selected databases and repositories, using all relevant terms and keywords identified in the previous step, according to the final bolenus phrase also defined in the previous step; 3 ) and, finally, in the third step, the reference lists of all included articles will be analyzed, in order to identify possible additional relevant studies.

It is intended to use as bibliographic reference management software Mendeley Desktop ${ }^{\circledR}$ version 1.19.4., to which the articles obtained in the research will be imported.

If necessary, contact will be made with the authors of the primary or secondary studies for further information or clarification.

- Study selection - The review process will consist of two levels of screening of the studies obtained: (1) a review of the title and abstract and (2) a review of the full text.

This selection process will be carried out independently by two researchers, where initially they will make the first selection of articles by reading the title and abstract, verifying that they meet the defined eligibility criteria. Any articles that are considered relevant by one or both researchers will be included in the full text review.

In the second screening phase, the two researchers will independently evaluate the full-text articles to determine if they meet the inclusion / exclusion criteria, thereby determining their inclusion in the review.

When disagreement occurs between the two researchers, full text articles will be reviewed again and further discussion will be conducted to ensure consistency between the researchers, and validation by a third researcher may be sought until consensus is reached.

If necessary, contact will be made with the authors of the primary studies to obtain further information or clarification on the data or even to obtain the article in full text format.

As for reference list studies, these will be identified, then the title and abstract will be analyzed, and then the full text will be analyzed. All who meet the eligibility criteria will be included in the review.

- Data extraction - Data will be extracted from full-text articles that meet the inclusion criteria, using an instrument created by the reviewers (Appendix I), following the model proposed by the Joanna Briggs Institute ${ }^{17}$ and aligned with the objective and the questions of the review. Data to be extracted from the studies include author(s), year of publication, country, study objective(s), study design, participants, characteristics of the programs/interventions implemented, data collection instruments used, main results and barriers/facilitators.

This process of extracting data from full text articles will be carried out by two independent researchers using the instrument created for this purpose (Appendix I). Discrepancies in this process of data extraction will be discussed between the two researchers until consensus is reached and /or with the use of a third researcher if necessary.

- Presentation of the results - The scoping review we pretend to carried out It aims to aggregate and summarize the results of the literature on programs/interventions to promote mental health literacy in 
adolescents in the school context, as well as to present an overview of the research developed in this area, so that the quality analysis of the articles individually is not an objective at this time and as such this assessment will not be made in this review.

The data obtained in the previous step will be compiled into a single table using Microsoft Excel ${ }^{\circledR}$ software for validation and coding.

It is intended to use a PRISMA flowchart to report the final numbers of the study selection process, as well as tables and graphs to present the main results of this review in a more visual way, as appropriate.

The main results of this scoping review will be summarized through a narrative description of the themes that emerge from the extracted data. Data will be discussed in the light of relevant theories, possible gaps found in the literature will be addressed and, consequently, suggestions will be made for future nursing research studies in the areas that require further investigation. 


\section{REFERENCES}

1. Biddle L, Donovan J, Sharp D, Gunnell D. Explaining non-help-seeking amongst young adults with mental distress: A dynamic interprative modelo of illness behaviour. Sociology of Health and Illness. 2007 Aug 30; 29(7): 983-1002.

2. Gulliver A, Griffiths K, Christensen H. Perceived barriers and facilitators to mental health help-seeking in young people: a systematic review. BMC Psychiatry. 2010 Dec 30; 10: 1-9.

3. Xavier M, Baptista H, Mendes J M, Magalhães P, Caldas-de-Almeida J M. Implementing the world mental health survey iniciative in Portugal - rationale, design and fieldwork procedures. Int J Ment Health Syst. $2013 \mathrm{Jul}$ 9; 7(1): 19.

4. World Health Organization [WHO]. The World Health Report 2001 - New Understanding, New Hope [Internet]. Switzerland: World Health Organization; 2001 [cited 2019 Set 20]. 178 p. Available from: https://www.who.int/whr/2001/en/whr01_en.pdf?ua=1

5. Kelly C, Jorm A, Right A. Improving mental heath literacy as a strategy to facilitate eraly intervention fou mental disorders. Med J Aust. 2007 Oct 1; 187(S7): S26-30.

6. World Health Organization [WHO]. Adolescent mental health: mapping actions of nongovernmental organizations and other international development organizations [Internet]. Switzerland: World Health Organization; 2012 [cited 2019 Set 29]. 49p. Available from: https://apps.who.int/iris/handle/10665/44875

7. Pedro A, Amaral O, Escoval A. Literacia em saúde, dos dados à ação: tradução, validação e aplicação do European Health Literacy Survey em Portugal. Revista Portuguesa de Saúde Pública. 2016 Out 19; 34(3): 259-275.

8. Medina M. Abrir Espaço à Saúde Mental - Estudo piloto sobre conhecimentos, estigma e necessidades relativas a questões de saúde mental, junto de alunos do 9. ano de escolaridade [master's thesis on the Internet]. Porto (Portugal): Universidade Católica Portuguesa, Faculdade de Educação e Psicologia (Centro Regional do Porto); 2013 [cited 2018 Oct 10]. 164 p. Available from: https://repositorio.ucp.pt/bitstream/10400.14/16946/1/Dissertação\%20de\%20Mestrado Miriam\%20 Medina.pdf

9. Pedreiro A. Literacia em saúde mental de adolescentes e jovens sobre depressão e abuso de álcool [master's thesis on the Internet]. Coimbra (Portugal): Instituto Politécnico de Coimbra, Escola Superior de Tecnologia da Saúde; 2013 [cited 2018 Oct 10]. 83 p. Available from: https://comum.rcaap.pt/bitstream/10400.26/11070/1/ANA PEDREIRO.pdf

10. Jorm A, Korten A, Jacomb P, Christensen H, Rodgers B, Pollitt P. "Mental health literacy": a survey of the public's ability to recognize mental disorders and their beliefs about the effectiveness of treatment. Med J Aust. 1997 Feb 17; 166(4): 182-186.

11. Rosa A, Loureiro L, Sequeira C. Literacia em saúde mental de adolescentes: Um estudo exploratório. Revista Portuguesa de Enfermagem de Saúde Mental. 2014 Apr; (spe1): 125-132.

12. Lluch M T. Concepto de salud mental positiva: Factores relacionados. In: Fornes J., Gómez J. editors. Recursos y programas para la salud mental. Enfermería psicosocial II. 2008: 37-69.

13. World Health Organization [WHO]. Mental health action plan 2013-2020 [Internet]. Switzerland: World Health Organization; 2013 [cited 2019 Set 20]. 50p. Available from: https://www.who.int/mental_health/publications/action_plan/en/ 
14. Morgado T, Botelho M R. Intervenções Promotoras da literacia em saúde mental dos adolescentes: Uma revisão sistemática da literatura. Revista Especial da Sociedade Portuguesa de Enfermagem de Saúde Mental. 2014 Apr; (spe1): 90-96.

15. Arksey H, O'Malley L. Scoping studies: towards a methodological framework. International Journal of Social Research Methodology [Internet]. 2005 Feb 1 [cited 2019 Set 20]; 8(1): 19-32. Available from: https://doi.org/10.1080/1364557032000119616

16. Peterson J, Pearce P F, Ferguson L A, Langford C A. Understanding scoping reviews: Definition, purpose, and process. Journal of the American Association of Nurse Practitioners [Internet]. 2017 Jan [cited 2019 Set 20]; 29(1): 12-16. Available from: https://doi.org/10.1002/2327-6924.12380

17. Peters M D J, Godfrey C, Mclnerney P, Baldini Soares C, Khalil H, Parker D. (2017). Chapter 11: Scoping Reviews. In: Aromataris E, Munn Z, Editors. Joanna Briggs Institute Reviewer's Manual [Internet]. Adelaide: The Joanna Briggs Institute; 2017 [cited 2019 Set 29]. Available from: https://reviewersmanual.joannabriggs.org/

18. Tricco A C, Lillie E, Zarin W, O'Brien $\mathrm{K} \mathrm{K}$, Colquhoun $\mathrm{H}$, Levac $\mathrm{D}$, et al. PRISMA extension for scoping reviews (PRISMA-SCR): Checklist and explanation. Ann Intern Med [Internet]. 2018 Oct 2 [cited 2019 Set 29]; 169(7): 467-473. Available from: https://doi.org/10.7326/M18-0850 
APPENDIX I: Instrument for Extracting Data from Review Articles

\begin{tabular}{|c|c|}
\hline \multicolumn{2}{|r|}{ Scoping Review Details } \\
\hline Title & \\
\hline Goal(s) & \\
\hline Review questions & \\
\hline \multicolumn{2}{|r|}{ Inclusion criteria } \\
\hline \multicolumn{2}{|l|}{ Participants } \\
\hline \multicolumn{2}{|l|}{ Concept } \\
\hline \multicolumn{2}{|l|}{ Context } \\
\hline \multicolumn{2}{|l|}{ Types of Studies } \\
\hline \multicolumn{2}{|c|}{ Study and Program/Intervention Details } \\
\hline \multicolumn{2}{|c|}{$\begin{array}{l}\text { Detailed citation of the study } \\
\text { (author/s, date, title, journal, volume, } \\
\text { issue, pages) }\end{array}$} \\
\hline \multicolumn{2}{|l|}{ Country } \\
\hline \multirow{2}{*}{\multicolumn{2}{|c|}{$\begin{array}{l}\text { Goal(s) of the study } \\
\text { Types of Study }\end{array}$}} \\
\hline & \\
\hline \multicolumn{2}{|l|}{ Program/intervention name } \\
\hline \multicolumn{2}{|l|}{$\begin{array}{l}\text { Goal(s) of the } \\
\text { program/intervention }\end{array}$} \\
\hline \multicolumn{2}{|l|}{$\begin{array}{l}\text { Participants of the } \\
\text { program/intervention }\end{array}$} \\
\hline \multicolumn{2}{|l|}{ Implementation context } \\
\hline \multicolumn{2}{|l|}{$\begin{array}{l}\text { Duration and frequency of } \\
\text { the program/intervention }\end{array}$} \\
\hline \multicolumn{2}{|l|}{$\begin{array}{l}\text { Description of the } \\
\text { program/intervention }\end{array}$} \\
\hline \multicolumn{2}{|l|}{ Evaluation instruments used } \\
\hline \multicolumn{2}{|l|}{ Main Outcomes } \\
\hline \multicolumn{2}{|l|}{ Barriers and Facilitators } \\
\hline \multicolumn{2}{|r|}{ Reference List } \\
\hline $\begin{array}{l}\text { Other studies of interest for } \\
\text { review indicated in study } \\
\text { reference list }\end{array}$ & \\
\hline
\end{tabular}

